\title{
A Review of Algorithms for Medical Image Segmentation and their Applications to the Female Pelvic Cavity
}

\author{
Zhen Ma ${ }^{\mathrm{a}}$, João Manuel R. S. Tavares ${ }^{1 \mathrm{a}}$, Renato Natal Jorge ${ }^{\mathrm{a}}$, T. Mascarenhas ${ }^{\mathrm{b}}$ \\ ${ }^{a}$ Faculdade de Engenharia da Universidade do Porto \\ Rua Dr. Roberto Frias, s/n \\ 4200-465 PORTO \\ PORTUGAL \\ Telephone: +351225081487 Fax: +351225081445 \\ Email: \{zhen.ma, tavares, rnatal\}@fe.up.pt \\ ${ }^{b}$ Faculdade de Medicina da Universidade do Porto \\ Al. Prof. Hernâni Monteiro, s/n \\ 4200 - 319 PORTO \\ PORTUGAL \\ Email: tqc@sapo.pt
}

${ }^{1}$ Corresponding author. Email: tavares@fe.up.pt 


\title{
A Review of Algorithms for Medical Image Segmentation and their Applications to the Female Pelvic Cavity
}

\begin{abstract}
This paper aims to make a review on the current segmentation algorithms used for medical images. Algorithms are classified according to their principal methodologies, namely the ones based on thresholds, the ones based on clustering techniques and the ones based on deformable models. The last type is focused due to the intensive investigations on the deformable models that have been done in the last few decades. Typical algorithms of each type are discussed and the main ideas, application fields, advantages and disadvantages of each type are summarized. Experiments that apply these algorithms to segment the organs and tissues of the female pelvic cavity are presented to further illustrate their distinct characteristics. In the end the main guidelines that should be considered for designing the segmentation algorithms of the pelvic cavity are proposed.
\end{abstract}

Keywords: Bioengineering, Biomedical Engineering, Medical Imaging, Algorithms Review, Thresholding Techniques, Clustering Techniques, Deformable Models, Female Pelvic Cavity. 


\section{Introduction}

The high resolution images produced by the modern imaging modalities offered to the medical doctors with multi-orientation views and much more details which have considerably assisted the clinical diagnosis and the following treatment. The first step of processing these images is to segment the desired organs or structures from image series. Usually to perform the manual segmentation medical technicians need to sketch the contours slice by slice using pointing devices such as a mouse or a trackball. This procedure is very timeconsuming and the results may suffer from intra-observer or inter-observer variability. In the past few decades, many algorithms have been proposed to perform the computer aided segmentation. The incorporations with modern mathematical and physical techniques have greatly enhanced the accuracy of the segmentation results. Compared with the algorithms for common images, the ones for medical applications need more concrete background. Information used for medical image processing comes not only from image appearances but also from imaging devices and doctors' professional knowledge. A prior knowledge such as the imaging environment or structures' biomechanical behavior can be crucial information for designing an effective algorithm, especially when the images are influenced by noises or partial volume effects (Zaidi, 2005). Also, the appearances of the same organs or structures may vary in different slices and imaging modalities and therefore, may need distinctive segmentation algorithms. For example, high density structures such as bones and calcified plaque have high Hounsfield Unit (HU) values in CT images and can be easily segmented, while they have much lower appreciable features in MR images so that the segmentation algorithm may not be trivial.

In the following sections, segmentation algorithms are classified into three main types based on their principal techniques, specifically the ones based on threshold, the ones based on clustering techniques and the ones based on deformable models. The main ideas, application fields, advantages and disadvantages of each type

are discussed and summarized. Each type is described using their typical algorithms and illustrated by the experimental results after these algorithms have been applied to the pelvic cavity. As it is common that multiple segmentation techniques are used in one algorithm, definite classifications of certain algorithms may not be feasible. The general principle of classification in this paper is the most appreciable characteristics of an algorithm.

The motivation for focusing on the segmentation of the pelvic cavity originates from the recognition of the high prevalence of pelvic floor (PF) disorders. Pelvic floor disorders include a group of conditions that affect adult women including pelvic organ prolapse, urinary incontinence, faecal incontinence, and other sensory 
and emptying abnormalities of the lower urinary and gastrointestinal tracts. The fact that women will be living longer and are more often attending to pelvic floor disorders makes its study urgent, in particular due to its high complexity and societal impact. It is estimated that $30 \sim 50 \%$ of woman in Europe and the USA are affected by urinary incontinence (Buchsbaum, et al., 2005). Over 20\% of French suffer from obstructed defecation (Siproudhis, et al., 2006). About 15\% of population of New Zealand and 2.2 6.9\% of population in the USA suffer from fecal incontinence (Drossman, et al., 1993; Nelson, et al., 1995; Macmillan, et al., 2004). The importance of developing a method to visualize, measure and model the dynamic responses of the pelvic structures has been more and more recognized. A real understanding of the pathophysiology of pelvic floor disorders is still lacking.

CT and MR images are widely used modalities for the study of the pelvic cavity. MR images are more preferred in the static study of pelvic cavity because CT images usually contain large amount of noises and have low spatial resolution of soft tissues. A successful segmentation of 2D image slices can greatly facilitate the later reconstruction of 3D pelvic cavity model which can help doctors understand its mechanism and establish a more objective basis for the diagnoses and the evaluation of the clinical treatments. The complex anatomical structures and the inter-connectivity between organs make the segmentation difficult to perform. Consequently, there is no effective algorithm for this task. Segmentation of pelvic cavity includes sketching bladder, urethra, vagina, rectum, obturator internus and levator ani. In this paper we do not intend to propose a new algorithm but use this example to illustrate the different characteristics of algorithms and make a discussion on their possible applications to this area. Furthermore, the main guidelines that an effective algorithm should adopt for a successful segmentation of the pelvic cavity are indicated.

The paper is organized as follows: In the next section, a review on the segmentation algorithms is made and their characteristics are illustrated through experiments on the pelvic cavity; In the third section, the advantages and disadvantages of each type are summarized; In the last section, some conclusions are summarized and the guidelines of the segmentation algorithms for the pelvic cavity are presented.

\section{Algorithms Review}

In this section we present a review based on the principal features of the algorithms, classifying them into three types as referred in Section 1. Due to the backgrounds and requirements for each task are different, a survey on a concrete application is beyond the scope of this paper. Instead, we extract the common features of 
each type, discuss and summarize their advantages and disadvantages using typical algorithms. Applications of each type to the pelvic cavity are illustrated to further state their characteristics.

\subsection{Algorithms based on thresholds}

Traditionally algorithms of this type make the premise that the interested structures or organs have distinctive quantifiable features such as the image intensity or the gradient magnitude. The procedure of segmentation is to search for the pixels whose values are within the ranges defined by the thresholds. Thresholds used in these algorithms can be selected manually or automatically. Manual selection needs a priori knowledge and sometimes trial experiments to find the proper threshold values while the latter way combines the image information to get the adaptive threshold values automatically. For example, the Otsu's method (Otsu, 1979) obtains the threshold values using image histogram. According to the information used to define the threshold values, algorithms can be further classified to edge-based ones, region-based ones and hybrid ones.

Threshold values in the edge-based algorithms are related with the edge information as structures are depicted by edge points. Wavelets transform (Chui, 1993) and common edge detection algorithms such as Canny edge detection (Canny, 1986), Sobel edge detection and Laplacian edge detection (Davis, 1975) belong to this type. Algorithms try to find edge pixels meanwhile eliminate the noise influence. For example, Laplacian edge detection uses the second derivation information of the image intensity; Canny edge detector uses the gradient magnitude to find the potential edge pixels and suppresses them through non-maximal suppression and hysteresis thresholding. Since the operations of the algorithms are based on pixel intensities, the detected boundaries consist of discrete pixels and therefore, may be incomplete or discontinuous. It is then necessary to apply post-processing techniques like morphological operations to connect the breaks or eliminate the holes. Due to noise influence and partial volume effect, the edges of organs or structures in medical images are usually not clearly defined. Therefore, algorithms based on threshold are seldom used alone but instead as an efficiency pre-processing step for the later segmentation (Andreao and Boudy, 2007; Qin et al, 2007).

The ideas of region-based algorithms come from the observation that quantifiable features inside a structure tend to be homogeneous. Algorithms aim to search for the pixels with similar feature values. Region growing algorithms (Adams, 1994; Pohle, 2001; Yi and Ra, 2001; Pan and Lu, 2007) are typical examples of this type. The searching rules are different among algorithms. For example, a simple approach can be to choose the initial seeds and merge their neighbor pixels whose intensities are within the threshold values until all the intensities of the surrounded pixels are outside the pre-defined ranges; the rule of seeded region growing 
method (Adams, 1994) is to expand a seed region through merging the unallocated neighbor pixels which have the smallest intensity difference between the pixel and the region; the rule of unseeded region growing algorithms are almost the same as the seeded region growing except when the within-class variance is too large or too small regions will split or merge. To eliminate the dependence on initial seeds and make the algorithm automatically, statistical information and a priori knowledge can be incorporated to the algorithms (Pohle and Toennies, 2001; Dehmeshki, et al., 2003). For example, a homogeneity criterion was introduced in (Pohle and Toennies, 2001) which made the region growing algorithms adaptive for different locations of the initial seeds and achieved success in the segmentation of CT and MR images. However, these algorithms have difficulties to control the leakage or eliminate the influence of partial volume effect due to their reliance on intensity.

Information used in the hybrid algorithms combine different image cues to complete the segmentation. Typical examples are the watershed algorithms (Beucher, 1979; Vincent, 1991; Grau, 2004; Ng, et al, 2006; Hamarneh and $\mathrm{Li}, 2009$ ) which combine the image intensity with the gradient information and use the mathematical morphology operations to do the segmentation.

In the watershed algorithms, gray scale images are considered as reliefs and the gradient magnitude is treated as elevation. Watershed lines are defined to be the pixels with local maximum gradient magnitude and a region of the image is defined as the pixels enclosed by the same watershed line. The segmentation procedure is to construct watersheds during the successive flooding of the gray value relief. Due to the combination of diverse image information, watershed algorithms can achieve satisfied results and always produce a complete segmentation of an image. Nevertheless, watershed algorithms tend to present over-segmentation problems, especially when the images are noisy or the desired objects themselves have low signal-to-noise ratio appearances. Hybrid threshold-based algorithms usually incorporate other techniques to perform the segmentation (Haris, et al., 1998; Grau, et al., 2004; Hamarneh and Li, 2009). For example, in (Grau, et al., 2004) the marker imposition technique was used to combine a prior knowledge and successful experiments have been reported on the segmentation of the knee cartilage and white/gray matter in MR images. In (Hamarneh and Li, 2009) shape and appearance knowledge are used to improve the performance of watershed algorithm and C-Means algorithm is used to handle the over-segmentation problems.

Figure 1(a) presents a MR axial pelvic cavity image, from which the appreciable influences of partial volume effects (PVE) can be easily seen. The segmentation results of Canny edge detector, region growing algorithm and watershed algorithm are illustrated in Figure 1(b)-(d). The boundaries obtained by Canny edge detection algorithm are discontinuous due to the noises and PVE. Besides, the spatial relationships of edge points are 
not reflected hence most of the detected boundaries are incomplete or connected wrongly. For the region growing algorithm the boundary of bladder and vagina are well segmented, but the boundary of right obturator internus leaks; the outer boundary of vagina is discontinuous and leaks in the upward direction. The watershed algorithm gives a complete segmentation of the image. However, the over segmentation can be seen in the area between bladder and vagina because there are a lot of pixels with local maximums of gradient magnitude. Moreover, the levator ani and obturator internus are merged because of the influence of PVE at the joint place.

\subsection{Algorithms based on clustering techniques}

As structures in medical images can be treated as patterns, techniques from pattern recognition fields can be used to perform the segmentation. Clustering techniques are the most popular ones for the medical image segmentation. In the following part, two main members of these algorithms are reviewed: supervised classification algorithms and unsupervised classification algorithms.

Frequently used supervised classification techniques include k-nearest neighbor classifier, maximum likelihood algorithm, supervised artificial neural network, support vector machine, active shape model, and active appearance model. A training set is needed to extract the information of structures while its functions are different among algorithms.

The training phase of k-nearest neighbor classifier $(\mathrm{kNN})$ is to store feature vectors and class labels of the training samples. K nearest stored points are selected for each unlabelled point according to the point distance. The classification of an unlabelled point is then voted by the selected points, for example the most frequent class label appeared in the selected points. The training step of maximum likelihood (ML) algorithms is to identify the parameters used in the statistical models. ML algorithms assume that the pixel intensities are independent random variables with parameterized probability distributions, so the probability distribution of this mixture model is given by the multiplication of these parameterized probability functions. Parameters are then evaluated by maximizing the likelihood function of the mixture model. As the calculations are based on

probability, ML algorithms provide a soft segmentation. Application of kNN and ML algorithms can be found in (Sarti, et al., 2005; Vrooman, et al., 2006).

Supervised artificial neural networks (ANNs) are non-linear statistical data modeling tools and can be used to model complex relationship between input and output. Weights or parameters in different layers are updated after processing each sample to minimize the cost function defined by the features of structures. Typical 
examples of neural network include feedforward network and radial basis function network (Bishop, 2006). Support vector machines (SVMs) are also known as maximum margin classifiers because the algorithms try to find a hyperplane to maximize the margin between two classes. SVMs can simultaneously minimize the empirical classification error and maximize the geometric margin between classes. For ANNs and SVMs information extracted from the training set provides the features of structure in the form of weights or parameters that can be used for the later segmentation. Common applications of ANNs and SVMs are on the segmentation of cardiac images and brain images inside which the organs and tissues have comparably stable shapes and anatomical structures (Bezdek, et al., 1993; Alirezaie, et al., 1997; Wang, et al., 2001; Benamrane, et al., 2006). Further classification and detailed procedures of ANNs and SVMs can be found in (James, 1985; Mitchell, 1997).

Unsupervised classification techniques include C-means algorithm (CM), Fuzzy C-means (FCM) algorithm, Iterative Self-organizing Data Analysis Technique Algorithm (ISODATA) and unsupervised neural networks. These algorithms are also called clustering algorithms. Structure features are extracted from the classified points.

C-means algorithm is also called K-means algorithms, where $\mathrm{C}$ and $\mathrm{K}$ are the pre-defined number of clusters. Algorithm tries to minimize the intra-cluster variation through iterations. The unlabelled pixels are assigned to the nearest clusters basing on their distances to the cluster centroids, then the cluster centroid is updated and the pixels are re-assigned. Algorithm ends until all the pixels have fixed labels. ISODATA algorithm is similar to the C-means algorithm while the number of clusters is determined by the threshold defined in the merging and splitting procedure. Applications of these algorithms are commonly used for nuclear medicine and transmission image segmentation (Jacobs et al, 2000; Biswal et al, 2006; Tai and Song, 2007). For the medical applications Fuzzy C-means (FCM) algorithms that combine CM algorithm with fuzzy theory have more applications. The procedures of Fuzzy C-means (FCM) algorithm are the same as the ones of CM algorithm except FCM adds weights to the calculation of cluster centroids and point distance. FCM algorithms are fuzzy clustering techniques that can provide soft segmentations in the way that instead of classifying a pixel into a fixed cluster, the algorithm calculates the membership or possibility that it belongs to each cluster. A soft segmentation is preferred as the complex imaging conditions such as shading artifacts or partial volume effects have intrinsically determined the vagueness of the pixels. The performance of FCM algorithms can be improved through adding spatial influence in the objective function (Pham and Prince, 1999; Ahmed et al, 2002; Cai et al, 2007; Wang et al, 2008; Zhou et al, 2008) or using kernel techniques that can better transfer non-linear problem to linear problem (Zhang and Chen, 2003; Liao et al, 2008). For example, 
in (Ahmed et al, 2002) the objective function is modified by adding an influence term defined by the labels in the neighborhood pixels. In (Liao et al, 2008) the proposed algorithm used a kernel filter to map the data into a higher-dimensional feature space and then apply the FCM algorithm with strategies introduced in (Cai et al, 2007) to gain high computational efficiency. FCM algorithms are widely applied to the segmentation of MR images especially for the MR brain images (Clark et al, 1994; Mohamed et al, 1999; Wang et al, 2008).

Unsupervised neural networks are based on unsupervised learning which means the targets are the same as the inputs (Sarle, 1994). The weights in the classifiers are trained according to the learning rule. For example, the Hopfields neural network adopts the learning rule as winner-takes-all to decide the weights either to be 0 (zero) or 1 (one). A successful application of Hopfields neural network to CT and MR images can be seen in (Cheng, et al., 1996; Lin, et al., 1996). Other popular learning rules include Hebbian learning and competitive learning (Bishop, 2006). A review on unsupervised neural network learning procedures and their applications can be found in (Becker and Plumbley, 1996).

There are also algorithms that use other pattern recognition techniques. For example, image registration techniques such as template matching algorithms and atlas-guided algorithms are also frequently seen to be applied for medical image segmentation (Gindi, et al., 1993; Ginneken, et al., 2002; Akselrod-Ballin, et al., 2006). A prior knowledge is used in these algorithms to assist the segmentation. For example, in (AkselrodBallin, et al, 2006) prior probability knowledge of anatomic structures is incorporated into a MRI probabilistic atlas to extract the structure features, then a SVM is trained using the extracted information to finally segment the brain structures.

Figure 2 presents the segmentation result of CM algorithm and its refinement using Markov Random Field techniques (Kindermann and Snell, 1980). Four clusters are defined with the initial mean intensities: 25, 80, 150, and 213. With these parameters CM algorithm can correctly segment the boundary of bladder. Most of the pixels that represent a same organ are clustered into one group. However, the noise influences are also appreciable. The boundaries of vagina and right obturator ani are incomplete and the boundaries of rectum and left obturator ani leaked. The refined result in Figure 2(b) gives a smoother segmentation with less noise influence because spatial constraint is incorporated using MRF techniques. Both segmentation results are very sensitive to the selection of initial number of groups and their mean intensity values.

Algorithms based on clustering techniques can be applied to segment the levator ani muscles. A successful segmentation of levator ani muscles is critical for the later 3D reconstruction of pelvic cavity. The muscles should have a presumed anatomy if they are not severely damaged. This characteristic can be easily guaranteed using algorithms based on clustering techniques. A manual segmentation of these muscles is 
shown in Figure 2(c). However, one should notice that the appearances of levator ani in static images are usually highly textured and have considerable variances in different slices. To identify the correct boundaries professional knowledge such as anatomical structures or combing multi-view images are needed. Modifications should focus on incorporating the spatial relationship between these muscles and other structures.

\subsection{Algorithms based on deformable models}

Compared with the above two types, the ones based on deformable models are more flexible and can be used for complex segmentations. Algorithms treat the structure boundary as the final status of the initial contours. The procedure of these algorithms can be viewed as a modelling of curve evolution. According to the way that is used for tracking the moving contour, deformable models can be further classified to parametric models and geometric models.

\subsubsection{Parametric deformable models}

Parametric deformable models track the evolution through sampled contour points. Explicit tracking way has the advantage of high computational efficiency and allows for real-time applications. The moving equation for the contour can be derived through energy functional or defined directly through dynamic forces. A prior knowledge can be incorporated in the procedure of defining the energy functional, the initial conditions or the parameters. A typical energy functional includes the internal energy and the external energy. The internal energy aims to keep the regularity of the contour and is usually defined through the geometric properties of the contour such as length, area or curvature; the external energy attracts the contour to the boundary position and is defined by the image information. The definitions of external forces are the main differences between algorithms. Using calculus of variations, the Euler-Lagrange (E-L) equation of the energy functional with the internal forces and external forces can then be derived simultaneously. Since the definition of energy functional guarantee that its minimum is achieved when the contours are at the position of structure boundaries, the E-L equation states that the balancing equilibrium of the contour under external forces and internal forces is the right position of the structure boundary. Then the moving equation can be derived through adding a time variable to the E-L equation. 
The development of parametric deformable models has tight relationship with the snake method (Kass, et al., 1987) which is the first deformable model applied to the medical image segmentation. The original snake method used the tension and rigidity of the contour as the internal energy and the gradient magnitude as the external energy. However, the snake method is sensitive to the initial conditions. The moving contour may stop at places with local functional minimum or places where the gradient magnitude is too small so that the external force tends to be zero. Also, the explicit tracking has the difficulty of handling topological changes. Consequently, in order to get a correct segmentation the initial contour must have the same topology as the desired object and must be placed near to the object boundary so that the external forces are strong enough. The later proposed algorithms aimed to eliminate the dependence on the initial position and the noise influence. For example, in order to prevent the curves from shrinking or stopping on local minimum, Cohen (Cohen, 1991) added a balloon force to the external forces to make the contour inflate or deflate when the gradient field is weak. Xu and Prince (Xu and Prince, 1998) analyzed the reason why snake methods have poor convergence to boundaries with large curvatures and replaced the gradient field with the gradient vector field (GVF), which has a larger capture region and slowly changes away from the boundaries. Consequently, the dependence on initial positions is decreased meanwhile the field can attract the moving contour to the right position. In (McInerney and Terzopoulos, 1995) except for adding the balloon force, an interactive mechanism was developed that allows user to select control points in order to form a constraining force to influence the curve movement. Numerical comparisons (He, et al., 2008) have shown that these improved algorithms considerably decrease the influence of initial conditions and improve the segmentation results.

Parametric deformable models which incorporate statistical techniques are also popular. Typical examples include active shape model (Cootes, et al., 1994; Cootes, et al., 1995) and active appearance model (Cootes, et al., 2001). Training samples are used to extract the mean shape and define proper ranges of the parameters. After finding an approximate position of the new examples, active shape model (ASM) uses the edge information to move the shape points to better positions while the active appearance model (AAM) used the mean texture of each shape point to find a better position. The searching procedure is like the snake methods but the movements of shape points are constrained by the ranges of shape parameters which guarantee the similarity between the segmentation result and the training samples. This characteristic is very useful when the shape or topology of structures can hardly be identified from their appearances in the images. ASM and AAM have been applied widely in medical image segmentation and registration (Cootes, et al., 1994; Ginneken, et al., 2002; Beichel, et al., 2005; Vasconcelos and Tavares, 2008). 
Parametric deformable models are widely used in structure segmentation and 3D reconstructions. A system review can be found in (McInerney and Terzopoulos, 1996). However, the computational complexity such as parameterization of the contour, handling of topological changes and re-distribution of the contour points considerably restrict their applications.

\subsubsection{Geometric deformable models}

The geometric deformable models are based on the level set method (Osher and Sethian, 1988) which was initially proposed to handle the topological changes during the curve evolution. The main idea of the level set method is to implicitly embed the moving contour into a higher dimensional level set function and view the contour as its zero level set. Then instead of tracking the discrete contour points, one can track the zero level set of the level set function. The advantage of doing so is that the topological changes can be easily handled and the geometric properties of the contour can be implicitly calculated. Therefore, the computational complexity of geometric deformable models is decreased. Like in the parametric deformable models, speed functions should be defined properly to drive the contour to the right position. Malladi et.al (Malladi, et al., 1993; Malladi and Sethian, 1996) and Caselles et.al (Caselles, et al., 1997) applied the level set methods to the medical image segmentation. Malladi’s algorithms used the gradient information to define the speed function and add the curvature influence to keep the contour smooth. The function of Malladi's speed model is intuitive: when the contour moves to the structure boundary, the increase of the gradient magnitude decreases the speed value so that the evolution of the contour slows down. Then the evolution can be stopped after a time to gain the position of structure boundary. However, Malladi’s speed models suffered from the drawback of leakage due to their bare dependence on the gradient information. The stopping criterion should be selected carefully to make sure the contour stops at the right position. If the images are noisy or blurred, the contour may leak or shrink to disappear after long time evolution (Kichenassamy, et al., 1996; Siddiqi, et al., 1998; Suri, et al, 2002). To handle the leakage, the edge strength item (Kichenassamy, et al, 1996) and area forces item (Siddiqi, et al., 1998) were incorporated to improve the model.

Unlike Malladi's model, geodesic active contour algorithm (GAC) modeled the segmentation as an optimization problem of finding the minimal distance curve in the image. Like in the parametric deformable models, the moving equation of GAC is derived from an energy functional; the procedure of finding the optimal solution corresponds to the searching of the structure boundary. The moving equation is then obtained through the Euler-Lagrange equation. While instead of tracking the contour points, the contour is embedded in 
a level set function and therefore, the moving equation becomes a level set equation. The speed function in GAC does not have an intuitive meaning; instead the derivation of the moving equation comes from energy functional. Unlike in Malladi's models the equilibrium state of the moving contour guarantees that a long time computation will not lead to leakage.

Geodesic active contour algorithm shows a tight relationship between the parametric model and the geometric model. The introduction of the level set expression makes the algorithm flexible to handle the topological changes. GAC and the later improved geodesic active contour algorithms are widely applied to process the MR, CT and ultrasound images like to accomplish tumor detection and cardiac segmentation. For example, in (Leventon, et al., 2000) a priori statistical techniques and shape information was incorporated. The contours are post-processed with the priori shape knowledge through using statistical techniques such as maximizing a posterior (MAP) and principle component analysis (PCA). In (Paragios, 2002) the proposed algorithm combined the gradient vector flow field with GAC algorithm to eliminate the dependence of the initial conditions; also intensity distributions in the left ventricle and the distance between the interfaces of endocardium and epicardium are incorporated. The results are better than the original GAC algorithm.

Another popular geometric model is proposed by Chan and Vese (Chan and Vese, 1999; Chan and Vese, 2002). Chan-Vese's model is a simplified version of Mumford-Shah energy model. The algorithm extracts the desired object through simultaneously minimizing the intensity variations inside and outside the contour. The most appreciable advantage of Chan-Vese's model is that it can obtain a boundary of discrete points, which is quite useful when the interested objects are represented by discrete pixel clusters and have no clear definition of boundaries.

Most of the moving equations of the later proposed geometric deformable models are derived through the energy functional because a priori knowledge and other techniques can be easily incorporated to the energy functional. Algorithms based on geometric deformable models aim to eliminate the noise influence, prevent the leakage, enhance the accuracy and the efficiency, make the algorithms more automatic and less dependent on the initial conditions. In order to these goals be achieved, algorithms incorporated various cues such as the image intensity, prior knowledge of structures. More details of geometric deformable models can be found in (Niessen, et al., 1998; Suri, et al., 2002). A survey of algorithms that combine statistical techniques with level set methods can be found in (Cremers, et al., 2007).

The Figures 3 and 4 illustrate the segmentation results of Malladi's algorithm and the geodesic active contour algorithm. Compared with the former segmentation results, the structure boundaries are more regular and less influenced by the noises due to the smoothing items defined in the speed function. Nevertheless, small shape 
details such as the left bottom part of bladder are also erased. Proper stopping criterion should be defined for Malladi's algorithm, otherwise the moving contours may leak, like the one illustrated in Figure 3(b). In this case the partial volume effects decrease the stopping strength at the right bottom boundary of rectum; hence the contour leaked due to long time evolution. In Figure 4, GAC algorithm provides a good segmentation of obturator internus, rectum and vagina. The regulating effects of internal forces make the boundary shape more reasonable and less influenced by noises. Also, leakage will not happen after long time evolution.

Deformable models are promising for the segmentation of pelvic cavity because these models can easily incorporate with statistical information, priori knowledge and other techniques while using the curve evolution to find the optimal boundaries can provide a contour with regular geometric properties. One possible way is to incorporate the comparative distances between organs and use prior shape information to constrain the deformation of the moving contour.

\section{Discussion}

When the interested structures have distinctive quantifiable features, using threshold-based algorithms is effective. Procedures of these algorithms do not include complex operations and therefore, are computationally efficient. However, due to their dependence on threshold values, algorithms are sensitive to noise, and most of them are difficult to be applied to multi-channel images. As medical images usually suffer from noise and intensity inhomogeneity, the segmentation results of threshold-based algorithms are far from satisfaction. Consequently, these algorithms are seldom used alone. Instead they are often used by combing with a priori knowledge (Dehmeshki et al, 2003; Pan and Lu, 2007) or as an efficient pre-segmentation step (Andreao and Boudy, 2007; Qin et al, 2007).

Except for the training step of the supervised classification algorithms, algorithms based on clustering techniques are also computationally efficient. Structure information can be easily used in these algorithms. When the interested structures in medical images are regular and not much influenced by noises, using pattern recognition techniques sometimes can achieve better results (Bezdek et al, 1993; Becker and Plumbley, 1997).

If properly modeled, supervised classification algorithms can greatly enhance the segmentation accuracy, especially when the appearance of structures is blurred or influenced by noises. The lack of incorporating spatial characteristics can be solved through using MRF model (Held, et al., 1997; Flitti, et al., 2005). Unsupervised classification algorithms can be applied to the cases when there are few available segmentation samples or when the interested structures have large shape variations and have shown promise for medical 
image segmentation in the fields like the tumor detection in positron emission tomography (PET) imaging (Zaidi, 2005). However, pattern recognition models are also sensitive to noise. Results of these algorithms depend on the initial conditions. For the supervised algorithms, the segmentation results depend on the size of the training sets and the correctness of the segmentations in samples. For the unsupervised algorithms, the number of clusters, the position of initial points and the parameters used in the model should be properly defined. The applications of both supervised algorithms and unsupervised algorithms are constrained because of the large shape variations of organs and structures in medical images.

Due to the advantages of being able to handle structures with complex topology, easy to incorporate with another techniques, sub-pixel accuracy, noise insensitive and intuitive interaction mechanisms, the deformable models are intensively investigated in the last few decades. Parametric deformable models have high computational efficiency and can easily incorporate a priori knowledge (McInerney and Terzopoulos, 1996). However, these models cannot naturally handle topological changes and are sensitive to initial conditions. Geometric deformable models have the advantage of naturally handling the topological changes and are widely studied for medical segmentation (Niessen et al, 1998). While using a parametric model or geometric model depends on the concrete segmentation task. The topological flexibility is not always a desirable feature under some applications (Han, et al., 2003; Guyader and Vese, 2008). In general, when structures have large shape variety or complicated topology, geometric deformable models are preferred; when the interested structures have open boundaries or the structures are thin or the algorithms need real-time operations, parametric models are preferred.

Deformable models in medical image segmentation have shown promising results and may continue to be focused in the next few years. Nevertheless, the deformable models also have disadvantages. Algorithms usually contain certain number of parameters. Parameters must be selected properly to get a satisfied result while this is usually a time-consuming task.

\section{Conclusions}

As pointed out in Section 1, most of the algorithms combine multiple segmentation techniques and use diverse image cues to improve the segmentation results. Therefore, a definite classification of an algorithm may be infeasible. In this paper, current algorithms are classified into three types and their respective characteristics are summarized. Applications of the current algorithms to segment the organs contained in pelvic cavity were 
illustrated. These examples were also used to a further state the distinct characteristics of different types of algorithms. From the discussions one can see that each segmentation algorithm category has its suitable application fields.

For the segmentation of the pelvic cavity as some organs like the bladder can be successfully segmented using the current algorithms, its information should be combined with the relative position of organs or a 3D atlas and other image cues to segment the objects that are influenced by partial volume effect or intensity inhomogeneity. To perform this applying the algorithms based on deformable models can be a good choice. For structures such as the pelvic floor muscles whose boundaries cannot be defined barely through the image appearance, a priori shape model and a restriction on shape variations are needed. These tasks can be better fulfilled using the algorithms based on clustering techniques. Pixels with special features such as the edge points can be used to identify the boundary position or define the energy functional. The searching of these pixels can then be done by the efficient algorithms based on thresholds. In conclusion, for a concrete segmentation task in medical images, the application background and practical requirements such as accuracy of segmentation, computational complexity and interactive ability should all be considered in the design of algorithms.

\section{Acknowledgment}

This work was partially done in the scope of the projects "Segmentation, Tracking and Motion Analysis of Deformable (2D/3D) Objects Using Physical Principles" and "BIOPELVIC-Study of Female Pelvic Floor Disorders", with references POSC/EEA-SRI/55386/2004 and PTDC/SAU-BEB/71459/2006, financially supported by Fundação para a Ciência e a Tecnologia of Portugal. The authors would like to thank the anonymous reviewers for their valuable suggestions.

\section{References}

Adams R, Bischof L. 1994. Seeded Region Growing. IEEE Trans. on Pattern Anal. Mach. Intelligence. 16(6):641-647.

Ahmed MN, Yamany SM, et al. 2002. A Modified Fuzzy C-means Algorithm for Bias Field Estimation and Segmentation of MRI Data. IEEE Trans. Med. Imaging. 21(3):193-199. 
Akselrod-Ballin A, Galun M, et al. 2006. Atlas Guided Identification of Brain Structures by Combining 3D Segmentation and SVM Classification. Paper presented at: MICCAI 2006. Proceeding of the 9th International Conference on Medical Image Computing and Computer Assisted Intervention; Copenhagen, Denmark.

Alirezaie J, Jernigan ME, Nahmias C. 1997. Neural Network-Based Segmentation of Magnetic Resonance Images of the Brain. IEEE Trans. on Nuclear Science. 44(2):194-198.

Andreao RV, Boudy J. 2007. Combining Wavelet Transform and Hidden Markov Models for ECG Segmentation. EURASIP Journal on Applied Signal Processing. (1):95-95.

Becker S, Plumbley M. 1996. Unsupervised Neural Network Learning Procedures for Feature Extraction and Classification. Journal of Applied Intelligence. 6(3):185-203.

Beichel R, Bischof H, et al. 2005. Robust Active Appearance Models and Their Application to Medical Image Analysis. IEEE Trans. on Med Img. 24(9):1151-1169.

Benamrane N, Aribi A, and Kraoula L. Fuzzy Neural Networks and Genetic Algorithms for Medical Images Interpretation. Paper presented at: GMAI 2006. Proceedings of Geometric Modeling and Imaging; London, UK.

Beucher S, Lantuéjoul C. 1979. Use of Watersheds in Contour Detection. Proceeding of International Workshop on Image Processing, Real-Time Edge and Motion Detection/ Estimation. Rennes, France.

Bezdek JC, Hall LO, and Clarke LP. 1993. Review of MR Image Segmentation Techniques Using Pattern Recognition. Med. Phys. 20(4):1033-1048.

Bishop CM. 2006. Pattern Recognition and Machine Learning. 1st ed. New York: Springer.

Biswal B, Shah N, et al. 2006. Automatic Segmentation of Pancreatic Images Using ISODATA Alorithm. Journal of Clinical Oncology, 2006 ASCO Annual Meeting Proceedings. 24(18):14149. 
Buchsbaum GM, Duecy EE, et al. 2005. Urinary Incontinence in Nulliparous Women and Their Parous Sisters. Obsete Gynecol. 106(6):1253-1258.

Cai WL, Chen SC, Zhang DQ. 2007. Fast and Robust Fuzzy C-Means Clustering Algorithms Incorporating Local Information for Image Segmentation. Pattern Recognition. 40(3):825-838.

Canny J. 1986. A Computational Approach to Edge Detection, IEEE Trans. Pattern Analysis and Machine Intelligence. 8(6):679-698.

Caselles V, Kimmel R, and Sapiro G. 1997. Geodesic Active Contours. Int. J. of Comp. Vision. 22(1):61-79.

Chan T, Vese LA. 1999. An Active Contour Model without Edges. Proceedings of the Int. Conf. Scale-Space Theories in Computer Vision; Corfu, Greece.

Chan T, Vese LA. 2002. Active Contour and Segmentation Models Using Geometric PDE’s for Medical Imaging. Geometric Methods in Bio-Medical Image Processing. Springer.

Cheng KS, Lin JS, and Mao CW. 1996. The Application of Competitive Hopfield Neural Network to Medical Image Segmentation. IEEE Trans. on Med. Img. 15(4):560-567.

Chui CK. 1993. An Introduction to Wavelets. SIAM Rev. 35(2):312-313.

Clark M, Hall L, et al. 1994. MRI Segmentation Using Fuzzy Clustering Techniques. IEEE Engineering in Medicine and Biology Magazine. 13(5):730-742.

Cohen LD. 1991. On Active Contour Models and Balloons. CVGIP: Image Understanding. 53(2):211-218.

Cootes TF, Hill A, et al. 1994. The Use of Active Shape Models for Locating Structures in Medical Images. Image and Vision Computing. 12(6):355-366. 
Cootes TF, Cooper D, et al. 1995. Active Shape Models - Their Training and Application. Computer Vision and Image Understanding. 6(1):38-59.

Cootes TF, Edwards GJ, Taylor CJ. 2001. Active Appearance Models. IEEE Trans. Pattern Analysis and Machine Intelligence. 23(6):681-685.

Cremers D, Rousson M, Deriche R. 2007. A Review of Statistical Approaches to Level Set Segmentation: Integrating Color, Texture, Motion and Shape. Int. J. of Comp. Vision. 72(2):195-215.

Davis LS. 1975. A Survey of Edge Detection Techniques, Comp. Graphics and Image Processing. 4(3):248270.

Dehmeshki J, Ye X, Costello J. 2003. Shape Based Region Growing Using Derivatives of 3D Medical Images: Application to Semiautomated Detection of Pulmonary Nodules. Paper presented at ICIP 2003. Proceedings of the 2003 International Conference on Image Processing; Barcelona, Spain.

Drossman DA, Li Z, Andruzzi E. 1993. U.S. Householder Survey of Functional Gastrointestinal Disorders: Prevalence, Sociodemography, and Health Impact. Dig. Dis. Sci. 38(9):1569-1580.

Flitti F, Collet C, Joannic-Chardin A. 2005. Unsupervised Multiband Image Segmentation Using Hidden Markov Quadtree and Copulas. Paper presented at: ICIP 2005, Proceedings of the 2005 International Conference on Image Processing; Genova, Italy.

Gonçalves PC, Tavares JM, Jorge RM. 2008. Segmentation and Simulation of Objects Represented in Images using Physical Principles. Computer Modeling in Engineering \& Sciences. 32(1):45-55.

Gindi G, Rangarajan A, Zubal G. 1993. Atlas-Guided Segmentation of Brain Images via Optimizing Neural Networks. Proceedings of the SPIE Biomedical Image Processing IV; San Jose, California, USA.

Ginneken BV, Frangi AF, et al. 2002. Active Shape Model Segmentation With Optimal Features. IEEE Trans. Med. Imag. 21(8):924-933. 
Grau V, Mewes AUJ., et al., 2004. Improved Watershed Transform for Medical Image Segmentation Using Prior Information. IEEE Trans. on Medical Imaging. 23(4):447-458.

Guyader CL, Vese LA. 2008. Self-Repelling Snakes for Topology-Preserving Segmentation Models. IEEE Trans. on Image Processing. 17(5):767-779.

Hamarneh G, Li XX. 2009. Watershed Segmentation Using Prior Shape and Appearance Knowledge. Image and Vision Computing. 27(1):59-68.

Han X, Xu CY, Prince JL. 2003. A Topology Preserving Level Set Method for Geometric Deformable Models. IEEE Trans. on Patter Analysis and Machine Intelligence. 25(6):755-768.

Haris K, Efstratiadis SN, et al. 1998. Hybrid Image Segmentation Using Watershed and Fast Region Merging. IEEE Trans. Image Processing. 7(12):1684-1699.

He L, Peng ZG, et al. 2008. A Comparative Study of Deformable Contour Methods on Medical Image Segmentation. Image and Vision Computing. 26(2):141-163.

Held K, Kops ER, et al. 1997. Markov Random Field Segmentation of Brain MR Images. IEEE Trans. on Medical Imaging. 16(6):878-885.

Jacobs MA, Knight RA, et al. 2000. Unsupervised Segmentation of Multiparameter MRI in Experimental Cerebral Ischemia with Comparison to T2, Diffusion, and ADC MRI Parameters and Histopathological Validation. JMRI. 11(4):425-437.

Kass M, Witkin A, Terzopoulos D. 1987. Snakes: Active Contour Models. Int. J. of Comp. Vision. 1(4):321331.

Kichenassamy S, Kumar A, et al. 1996. Conformal Curvatures Flows: From Phase Transitions to Active Vision. Arch. Rational Mech. Anal. 134(3):275-301. 
Kindermann R, Snell JL. 1980. Markov Random Fields and Their Applications. Rhode Island: American Mathematical Society.

Leventon M, Grimson W, Faugeras O. 2000. Statistical Shape Influence in Geodesic Active Contours. Paper presented at: CVPR 2000. Proceedings of the IEEE Conf. on Comp. Vision and Pattern Recognition; Hilton Head Island, South Carolina, USA.

Liao L, Lin TS, Li B. 2008. MRI Brain Image Segmentation and Bias Field Correction Based on Fast Spatially Constrained Kernel Clustering Approach. Pattern Recognition Letters. 29(10):1580-1588.

Lin JS, Cheng KS, and Mao CW. 1996. A Fuzzy Hopfield Neural Network for Medical Image Segmentation. IEEE Trans. on Nuclear Science, 43(4):2389-2398.

Lin Z, Jin J, and Talbot H. 2000. Unseeded Region Growing for 3D Image Segmentation. Paper presented at: VIP 2000. Proceeding of the Pan-Sydney Area Workshop on Visual Information Processing; Sydney, Australia.

Luo S, Zhang Q, et al. 2004. An Improved Moment-preserving Auto Threshold Image Segmentation Algorithm. Paper presented at: ICIA 2004. Proceedings of the Int. Conf. on Information Acquisition; Hefei, China.

Macmillan AK, Merrie AEH, et al. 2004. The Prevalence of Fecal Incontinence in Community-dwelling Adults: A Systematic Review of The Literature. Dis Colon Rectum. 47(8):1341-1349.

Malladi R, Sethian JA, Vemuri B. 1993. A Topology Independent Shape Modeling Scheme. Proceedings of the SPIE Conf. on Geometric Methods in Computer Vision II; San Diego, California, USA.

Malladi R, Sethian JA. Level Set and Fast Marching Methods in Image Processing and Computer Vision. Paper presented at: ICIP 96. Proceedings of the IEEE International Conference on Image Processing; Lausanne, Switzerland. 
McInerney T, Terzopoulos D. 1995. A Dynamic Finite Element Surface Model for Segmentation and Tracking in Multidimensional Medical Images with Application to Cardiac 4D Image Analysis. Computerized Medical Imaging and Graphics. 19(1):69-83.

McInerney T, Terzopoulos D. 1996. Deformable Models in Medical Image Analysis: A Survey. Medical Image Analysis. 1(2):91-108.

McInerney T, Terzopoulos D. 2000. T-snakes: Topology Adaptive Snakes. Medical Image Analysis. 2000, 4(2):73-91.

Mike J. 1985. Classification Algorithms. 1st ed. New York: Wiley-Interscience.

Mohamed NA., Ahmed MN, Farag A. 1999. Modified Fuzzy C-mean in Medical Image Segmentation. Paper presented at: ICASSP 99. Proceedings of the 20th Annual International Conference on Acoustics, Speech, and Signal Processing. Phoenix, Arizona, USA.

Nelson R, Norton N, et al. 1995. Community Based Prevalence of Anal Incontinence. JAMA. 274(7):559561.

Ng HP, Ong SH, et al. 2006. Medical Image Segmentation Using K-Means Clustering and Improved Watershed Algorithm. Paper presented at: SSIAI 2006. Proceedings of the IEEE Southeast Symposium on Image Analysis and Interpretation; Denver, Colorado, USA.

Niessen WJ, Romeny EM, Viergever MA. 1998. Geodesic Deformable Models for Medical Image Analysis. IEEE Trans. Med. Img. 17(4):634-641.

Osher S, Sethian J. 1988. Fronts Propagating with Curvature-dependent Speed: Algorithms Based on Hamilton-Jacobi Formulations. J. Comp. Phys. 79(1):12-49. 
Otsu N. 1979. A Threshold Selection Method from Gray-level Histograms. IEEE Trans. Sys., Man., Cyber. 9(1):62-66.

Pan ZG, Lu JF. 2007. A Bayes-Based Region-Growing Algorithm for Medical Image Segmentation. Computing in Science \& Engineering. 9(4):32-38.

Paragios N, 2002. A Variational Approach for the Segmentation of the Left Ventricle in Cardiac Image Analysis. International Journal of Computer Vision. 50(3):345-362.

Pham DL, Prince JL. 1999. An Adaptive Fuzzy C-means Algorithm for Image Segmentation in The Presence of Intensity Inhomogeneities. Pattern Recognition Letter. 20 (1):57-68.

Pohle R, Toennies KD. 2001. Segmentation of Medical Images Using Adaptive Region Growing. Paper presented at: MI 2001. Proceedings of the SPIE Medical Imaging 2001. San Diego, California, USA.

Qin XJ, Jiang JH, et al. 2007. Canny Operator Based Level Set Segmentation Algorithm for Medical Images. Paper presented at: ICBBE 2007. Proceedings of the $1^{\text {st }}$ International Conference on Bioinformatics and Biomedical Engineering; Wuhan, China.

Sarle WS. 1994. Neural Networks and Statistical Models. Paper presented at: SUGI 19. Proceedings of the $19^{\text {th }}$ Annual SAS Users Group International Conference; Dallas, Texas, USA.

Sarti A, Corsi C, et al., 2005. Maximum Likelihood Segmentation of Ultrasound Images with Rayleigh Distribution. IEEE Trans.Ultrasonics Ferroelectrics and Frequency Control. 52(6):947-960.

Siddiqi K, Lauriere YB, et al, 1998. Area and Length Minimizing Flows for Shape Segmentation. IEEE Trans. Image Processing. 7(3):433-43.

Siproudhis L, Pigot F, Godeberge P, et al. 2006. Defecation Disorders: A French Population Survey. Dis Colon Rectum. 49(2):219-227. 
Suri JS. 2000. Computer Vision, Pattern Recognition and Image Processing in Left Ventricle Segmentation: The Last 50 Years. Pattern Analysis \& Applications. 3(3):209-242.

Suri JS, Liu KC, et al. 2002. Shape Recovery Algorithms Using Level Sets in 2-D/3-D Medical Imagery: A State-of-the-Art Review. IEEE Trans.Information Technology in Biomedicine. 6(1):8-28.

Tai XY, Song WH. 2007. An Improved Approach Based on FCM Using Feature Fusion for Medical Image Retrieval. Paper presented at: FSKD 2007. Proceedings of the 4th International Conference on Fuzzy Systems and Knowledge Discovery; Hainan, China.

Mitchell T. 1997. Machine Learning. 1st ed. McGraw-Hill.

Vasconcelos MJM, Tavares JMRS. Methods to Automatically Built Point Distribution Models for Objects like Hand Palms and Faces Represent in Images. Computer Modeling in Engineering \& Sciences. 36(3):213241.

Vincent L, Soille P. 1991. Watersheds in Digital Spaces: An Efficient Algorithm Based on Immersion Simulations. IEEE Trans. Pattern Analysis and Machine Intelligence. 13(6):583-598.

Vrooman HA, Cocosco CA, et al. kNN-based Multi-spectral MRI Brain Tissue Classification: Manual Training versus Automated Atlas-based Training. Paper presented at: MI 2006. Proceedings of the SPIE Medical Imaging 2006; California, USA.

Wang S, Zhu WY, Liang ZP. 2001. Shape Deformation: SVM Regression and Application to Medical Image Segmentation. Paper presented at: ICCV 2001. Proceedings of the $8^{\text {th }}$ International Conference on Computer Vision; Vancouver, British Columbia, Canada.

Wang JZ, Kong J, et al. 2008. A Modified FCM Algorithm for MRI Brain Image Segmentation Using Both Local and Non-local Spatial Constraints. Computerized Medical Imaging and Graphics. 32(8):685-698. 
Xu CY, Prince JL. 1998. Snakes, Shapes, and Gradient Vector Flow. IEEE Trans. on Image Processing. 7(3):359-369.

Yi J, Ra JB. 2001. Vascular Segmentation Algorithm Using Locally Adaptive Region Growing Based on Centerline Estimation. Paper presented at: MI 2001. Proceedings of the SPIE Medical Imaging 2001; San Diego, California, USA.

Zaidi H. 2005. Quantitative Analysis in Nuclear Medicine Imaging. $1^{\text {st }}$ ed. New York: Springer.

Zhang DQ, Chen SC. 2003. Robust Image Segmentation Using FCM with Spatial Constraints Based on New Kernel-Induced Distance Measure. IEEE Trans. on System, Man and Cybernetics. 34(4):1907-1916.

Zhou HY, Schaefer G, et al. 2008. Anisotropic Mean Shift Based Fuzzy C-means Segmentation of Skin Lesions. Paper presented at: CSTST 2008. Proceedings of the 5th International Conference on Soft Computing as Transdisciplinary Science and Technology; Cergy-Pontoise, Paris, France. 


\section{FIGURE CAPTIONS}

Figure 1: (a) MR image of the pelvic cavity; (b) Canny edge detection; (c) Region growing algorithm: 1bladder; 2-vagina; 3-rectum; 4-levator ani; 5-obturator internus; (d) Watershed algorithm.

Figure 2: (a) C-means algorithm (number of groups: 4; initial mean values of intensity: 25, 80, 150, 213); (b) Refined by MRF; (c) Manual segmentation of levator ani muscles.

Figure 3: (a) Malladi's algorithm (annotations are the same as in Figure 1c); (b) Leakage due to over evolution using Malladi’s algorithm.

Figure 4: Geodesic active contour algorithm (annotations are the same as in Figure 1c). 


\section{FIGURES}

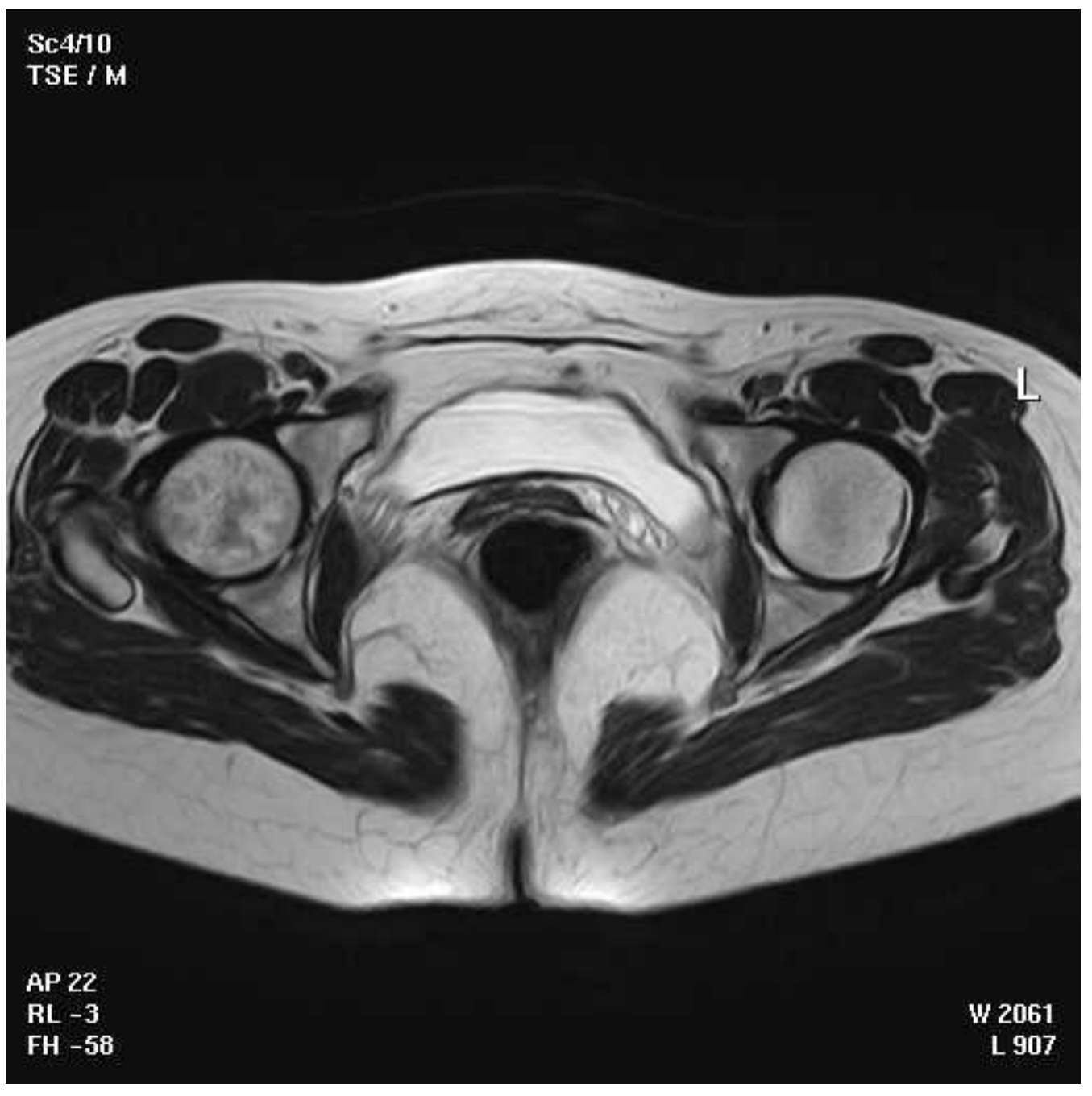

Figure 1a 


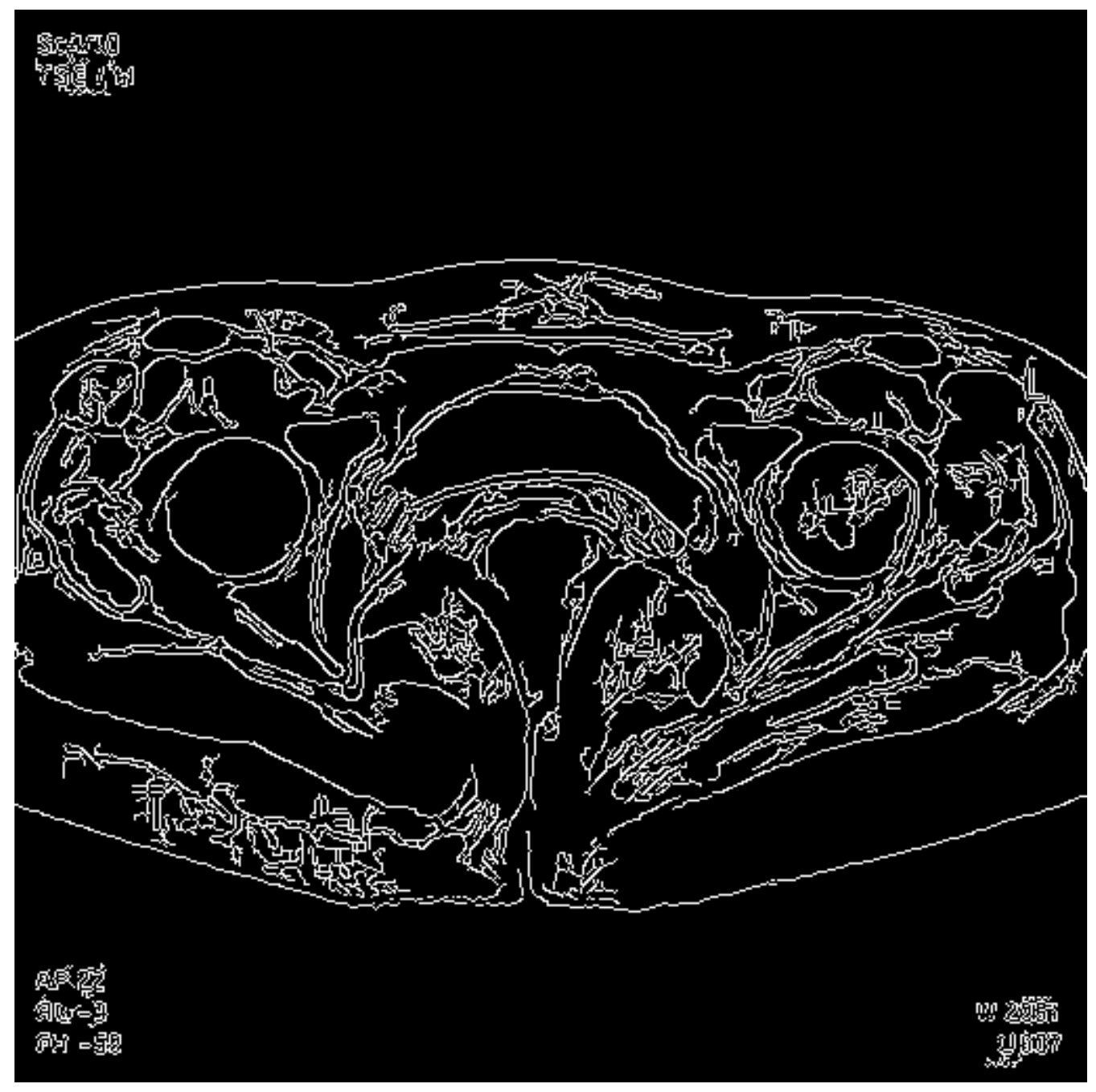

Figure 1b 


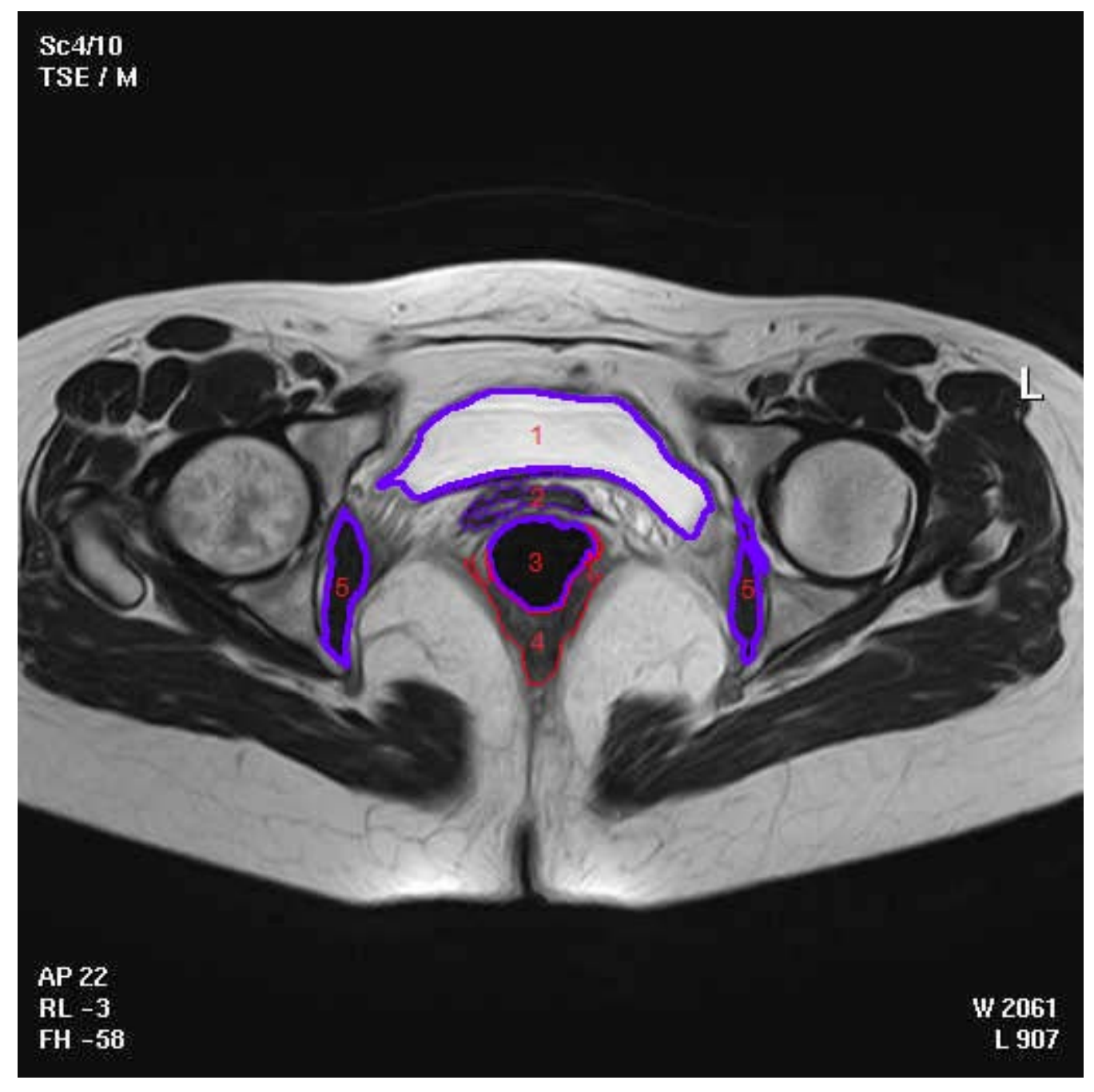

Figure 1c 


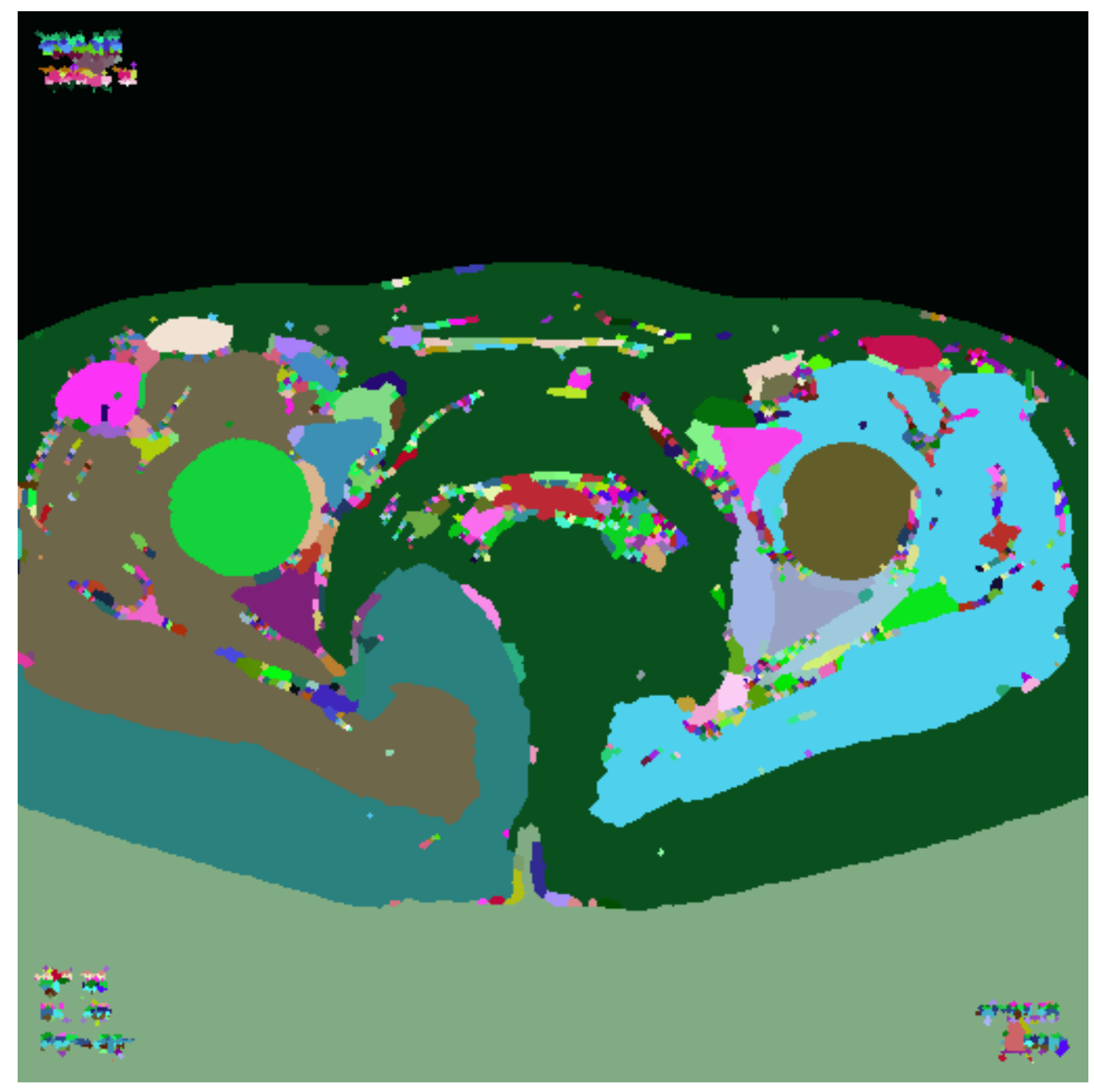

Figure 1d 


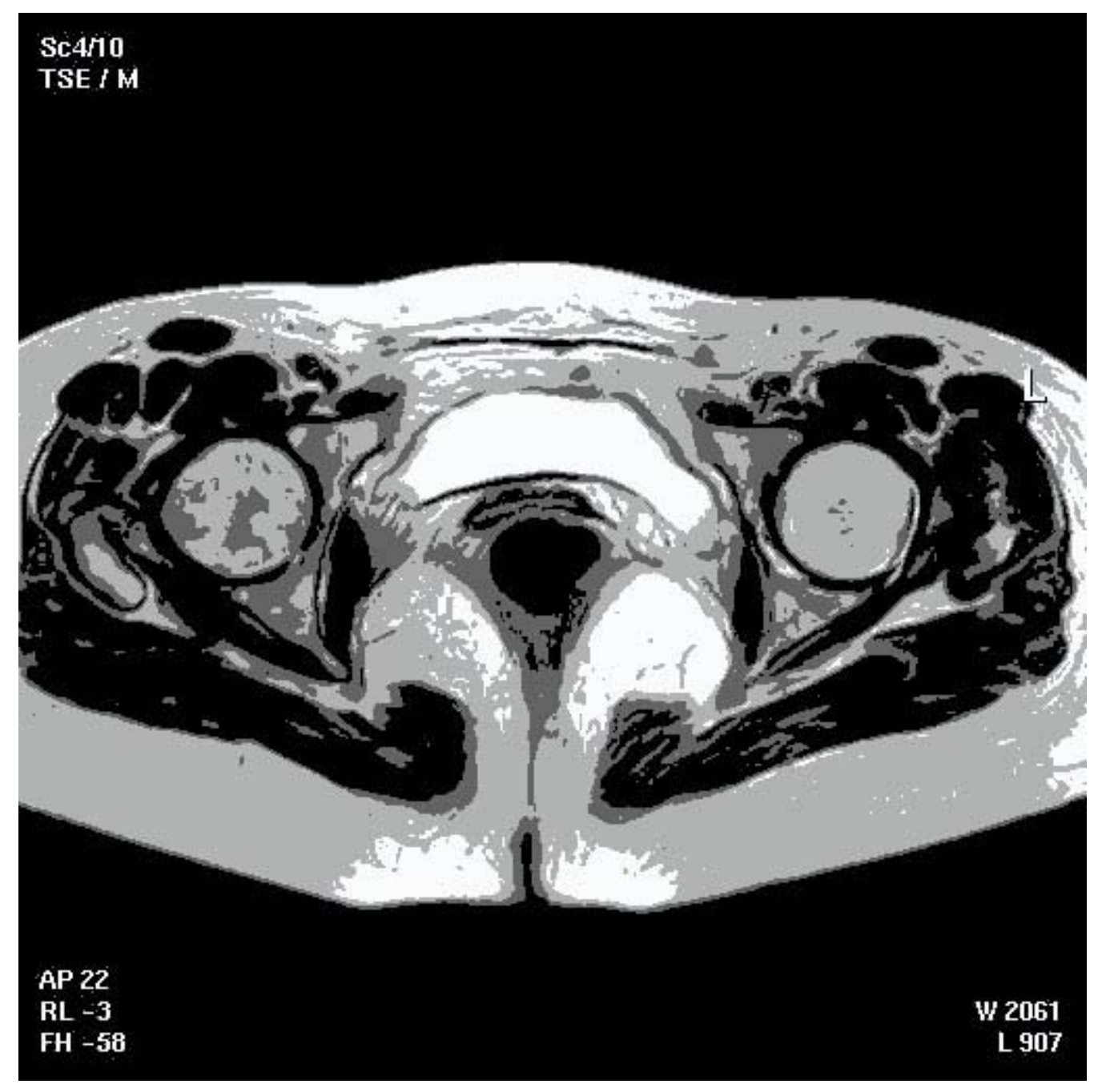

Figure 2a 


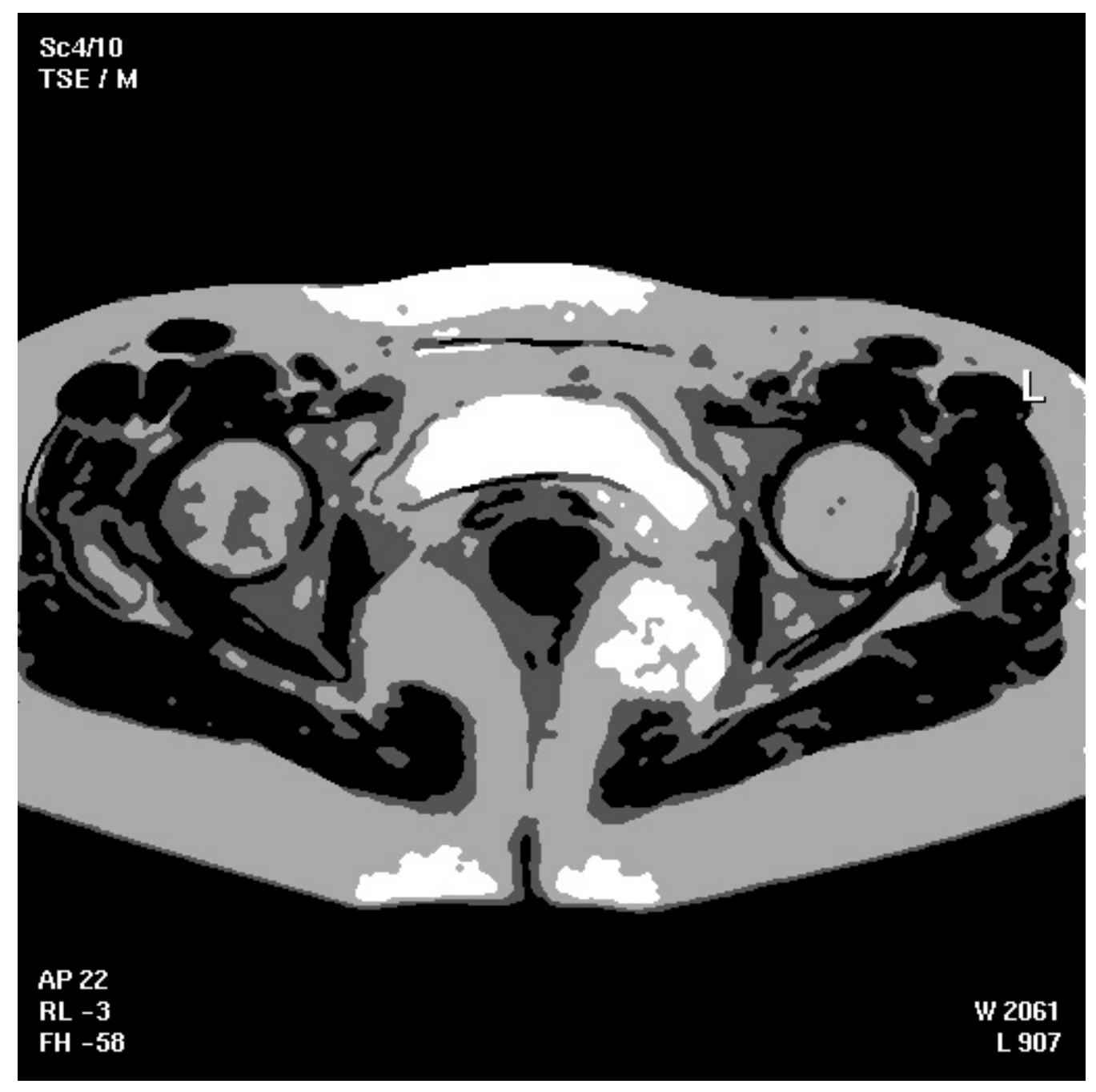

Figure 2b 


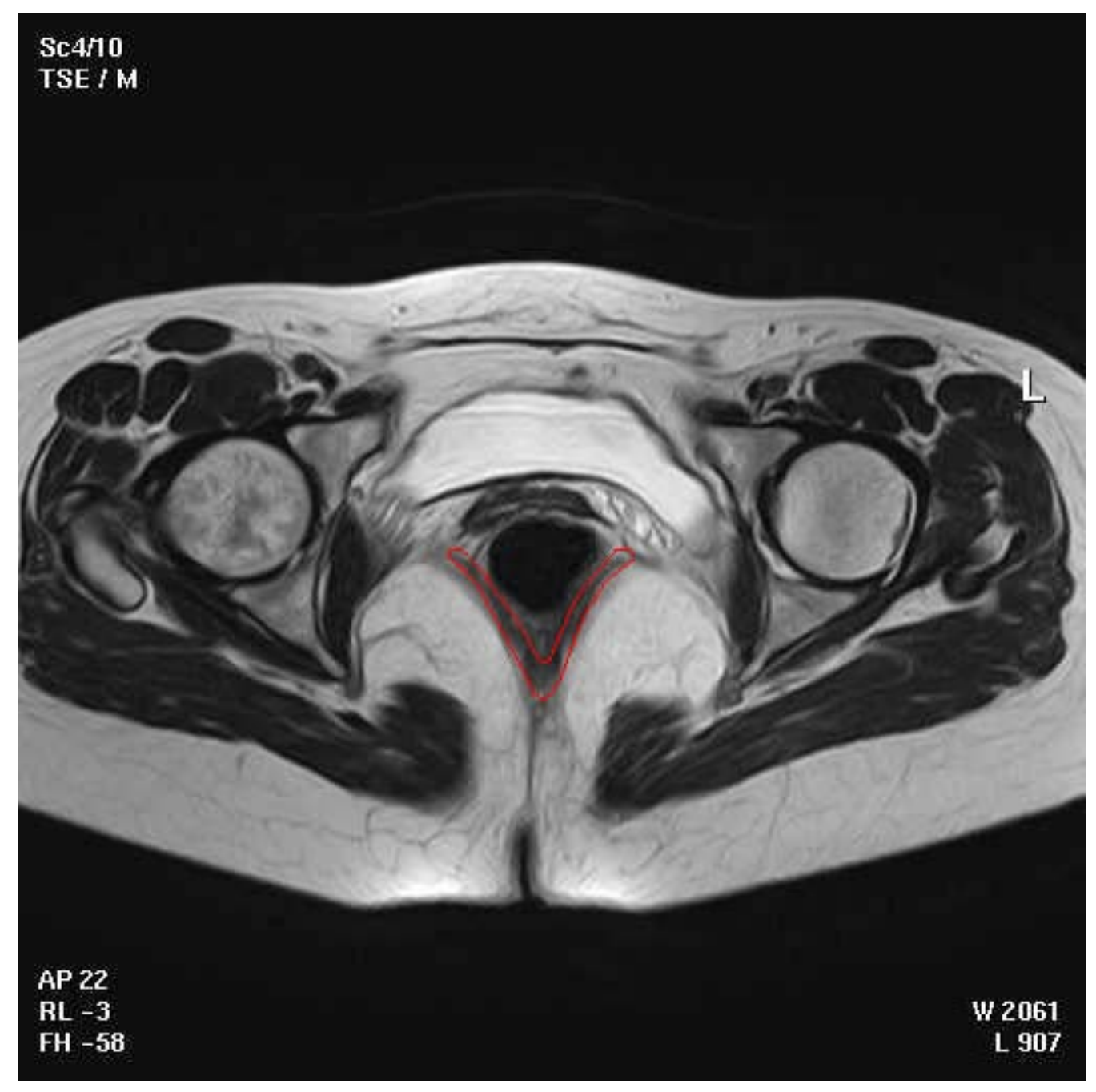

Figure 2c 


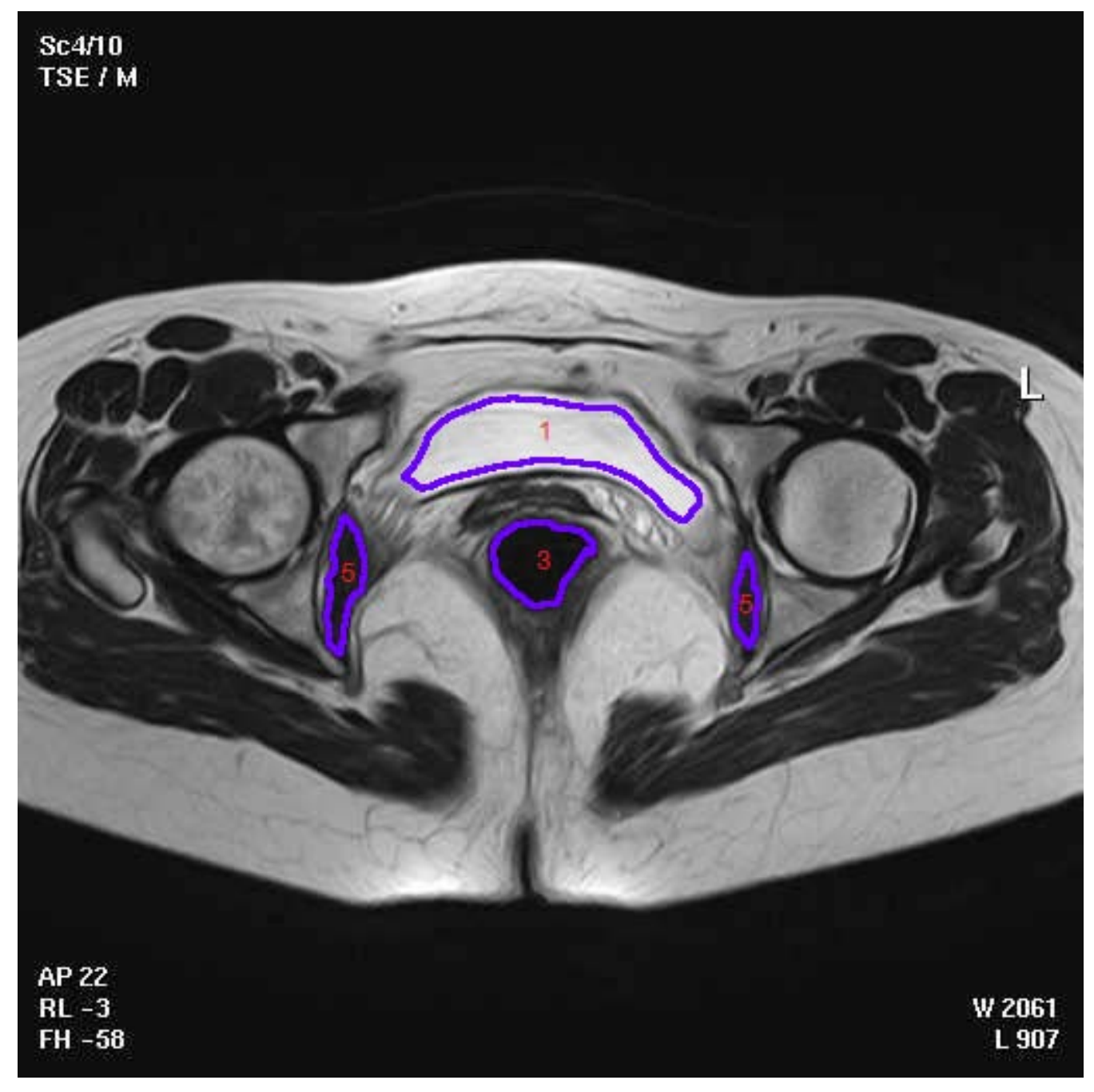

Figure 3a 


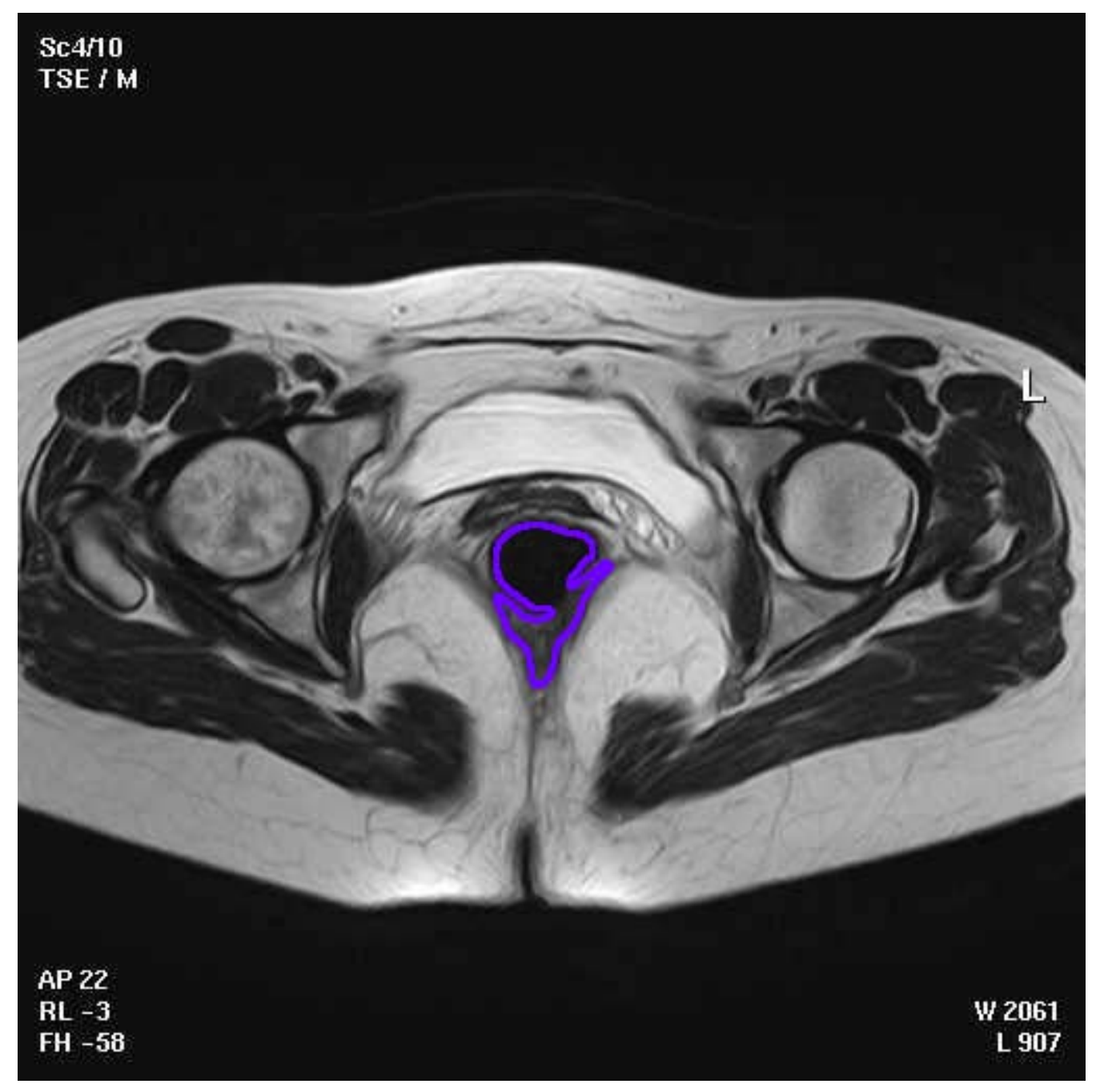

Figure 3b 


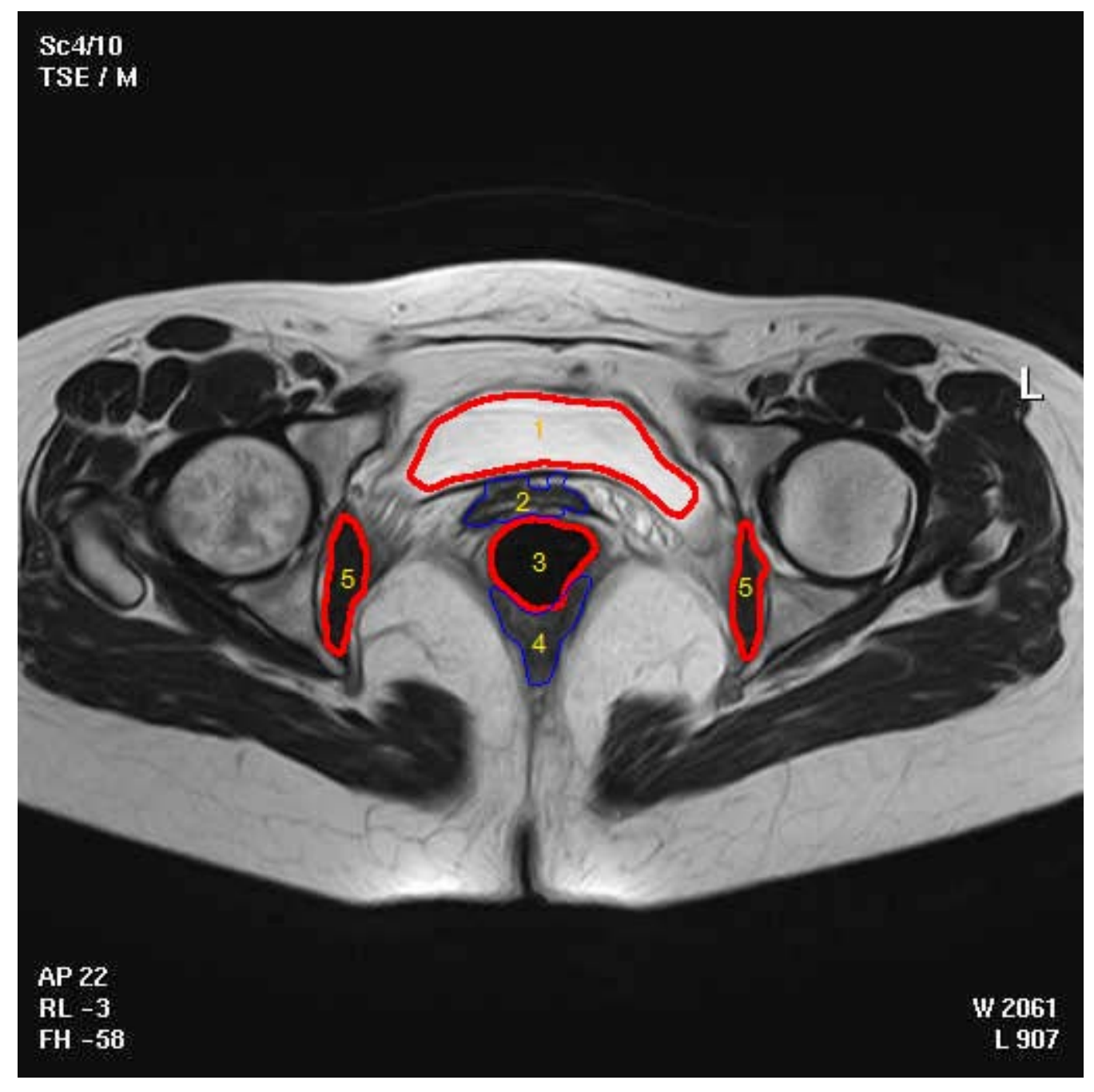

Figure 4 